\title{
¿El volumen medio plaquetario es un predictor de mortalidad en pacientes sépticos? Revisión de la literatura
}

\begin{abstract}
The mean platelet volume is a predictor of mortality in septic patients? Review of the literature
\end{abstract}
Jorge Luis Vélez ${ }^{1, a, b}$

RESUMEN

La sepsis es una de las causas más frecuentes que motivan el ingreso de pacientes al hospital y a las unidades de cuidados intensivos, tiene alto impacto en la morbilidad y mortalidad. Pese a los avances tecnológicos que han permitido mejorar el soporte de fallos orgánicos, no se ha logrado disminuir de forma importante sus complicaciones. Por ello es importante tener herramientas que sean predictoras de severidad en esta patología; los biomarcadores (procalcitonina, pro adrenomodulina, interleuquina 6), se han convertido en buenos índices de pronostico y evolución, sin embargo, su disponibilidad ya sea por el costo o existencia de reactivos en los distintos laboratorios no es constante; es por ello que buscamos con ésta revisión determinar si el uso del volumen medio plaquetario, un biomarcador reportado en el hemograma común y que se viene usando con relativo éxito en patologías cardiovascular, metabólica e inflamatoria/infecciosa, como un indicador de pronóstico de severidad y mortalidad.

PALABRAS CLAVE: Plaquetas, sepsis, biomarcadores, mortalidad. (Fuente: DeCS BIREME).

\section{SUMMARY}

Sepsis is one of the most common causes of hospital admission to intensive care units and has a high impact on morbidity and mortality. In spite of the technological advances that have allowed to improve the support of organic failures, it has not been possible to diminish of important form its complications. Therefore, it is important to have tools that are predictive of severity in this pathology, the biomarkers (procalcitin, proadrenomodulin, interleukin 6) have become good indicators of prognosis and evolution, however their availability either by cost or existence of reagents in the different laboratories is not constant; this is why we seek with this review to determine if determine if the use of mean platelet volume, a biomarker reported in the common hemogram and has been used with relative success in cardiovascular, metabolic and inflammatory / infectious pathology, as an indicator of prognosis of severity and mortality.

KEYWORDS: Platelets, sepsis, biomarkers, mortality. (Source: MeSH NLM).

\section{INTRODUCCIÓN}

La sepsis es una patología altamente prevalente a nivel mundial, pocas medidas han podido disminuir su mortalidad, exceptuando el inicio temprano de la antibioticoterapia y el adecuado soporte de los fallos orgánicos, la posibilidad de determinar la severidad de ésta entidad es importante y se han conseguido avances con escalas y marcadores fisiológicos (escala APACHE II), bioquímicos (procalcitonina, interleucina 6, proteína C reactiva) y anatómicos, sin embargo muchos de ellos no son asequibles a la práctica clínica

Unidad de Terapia Intensiva del Hospital Pablo Arturo Suárez - Universidad Central del Ecuador. Quito, Ecuador.

Médico Especialista en Medicina Crítica;

Master en Investigación Clínica y Epidemiología. 
rutinaria ya sea por falta de disponibilidad o alto costo para realizarlo.

En este sentido, un biomarcador anatómico, el volumen medio plaquetario (VMP) medido en fentolitros (fl), poco utilizado, pero reportado en los hemogramas rutinarios, ha dado un rendimiento pronóstico adecuado en estados inflamatoriostrombóticos-metabólicos como el infarto agudo de miocardio, la diabetes mellitus y la enfermedad cerebro vascular isquémica, en ésta última su incremento es un fuerte predictor de mal pronóstico (1).

En la sepsis el VMP se ha estudiado como marcador pronóstico, son varios los trabajos sobre este campo con resultados promisorios, su valor absoluto y el cociente del VMP y el recuento de plaquetas han resultado adecuados predictores de mala evolución; sin embargo, han sido realizados con pocos pacientes y no se ha establecido un valor de corte referencial.

Recientemente un metaanálisis con más de 2000 pacientes encontró útil su aumento de tamaño en el día 3 de evolución (2), sin embargo, la heterogeneidad de los estudios utilizados no permite que sus resultados sean categóricos.

Por esta razón es necesario plenamente trabajar sobre este tema, ya que el validar al VMP como biomarcador pronóstico en sepsis, significaría tener una herramienta práctica, costo-efectiva y rápidamente disponible.

En este trabajo el objetivo fue hacer una revisión de la literatura sobre la utilidad del volumen medio plaquetario como predictor de mortalidad en pacientes sépticos.

Se realizó una búsqueda de la literatura en las bases de datos PUBMED, LILACS, MEDLINE, con las palabras clave en inglés y español: mean platelet volume, sepsis, mortality.

\section{¿Qué es el VMP?}

El VMP es la medición geométrica del tamaño de las plaquetas y tiene una relación inversa con el número de éstas, es además un indicador de la activación plaquetaria (3).

No se cuenta con un punto de corte establecido porque los valores son cambiantes y varían de población en población de acuerdo con la técnica utilizada para medirlo y el tiempo de realización de la prueba; sin embargo, está establecido que los valores superiores a 9,5 fl se asocian con enfermedades que tienen como sustrato la inflamación, disfunción endotelial y un estado protrombótico (3).

En el pasado el uso del VMP se limitaba al diagnóstico de alteraciones plaquetarias inusuales (trombocitopenias congénitas o autoinmunes), sin embargo, en la modernidad ha adquirido gran importancia en patología clínica, siendo utilizado para determinar morbilidad y mortalidad en varias entidades nosológicas de diversa fisiopatología (infecciones, cardiovasculares, metabólicas).

Laelevación delVMPseasocia conotros marcadores de actividad plaquetaria, incluyendo el incremento en la agregación plaquetaria, aumento en la síntesis del tromboxano, liberación de $\beta$-tromboglobulina y aumento en la expresión y adhesión de moléculas (3).

Está demostrado que el incremento del VMP se asocia con mal pronóstico en pacientes con síndrome metabólico, diabetes mellitus, sepsis, enfermedades cardiovasculares, embolismo pulmonar, tabaquismo y enfermedades inmuno-inflamatorias (3).

\section{VMP y eventos trombóticos}

Un VMP elevado al ingreso del paciente con un evento trombótico agudo, así como su elevación en los días posteriores (los valores máximos de volumen plaquetario medio alcanzan sus valores al sexto día a partir del suceso cardiovascular), está asociado con aumento en la mortalidad posterior a un infarto agudo de miocardio y a re estenosis después de angioplastia coronaria. El volumen plaquetario medio es un factor de riesgo independiente de isquemia y muerte. La evidencia científica sugiere al volumen plaquetario medio como un marcador pronóstico útil en pacientes con enfermedad cardiovascular, y su elevación se relaciona con eventos y desenlaces cardiovasculares adversos (4).

En infarto agudo de miocardio un VMP superior a $9 \mathrm{fl}$ se relacionó con un aumento estadísticamente significativo del riesgo no ajustado del punto final combinado de muerte, insuficiencia cardíaca o episodios isquémicos postinfarto $(\mathrm{OR}=1,37$; $\mathrm{IC}$ del 95\%: $1,06-1,76, \mathrm{p}=0,01)(5)$.

Por un lado, el volumen plaquetario elevado se ha relacionado con pobres desenlaces en el evento vascular cerebral isquémico independientemente de 
otros parámetros, y por otro lado, la cuenta plaquetaria se encuentra significativamente disminuida en pacientes con evento vascular cerebral isquémico con pobre pronóstico (4).

\section{VMP y sepsis}

En sepsis, investigadores de la Klinikum St. Georg (Leipzig, Alemania) (6) realizaron un estudio con 191 pacientes (edad media 72 años, 38\% mujeres) con sepsis documentada. Los investigadores evaluaron prospectivamente el VMP al momento de la admisión, en el inicio de la sepsis, en el diagnóstico de sepsis, y durante el curso de la enfermedad, como un marcador para la predicción de los resultados. Estos datos fueron comparados con datos de 56 pacientes (edad mediana, 74 años, mujeres, 45,5\%) con sangrado agudo gastrointestinal superior e inferior (GI), que sirvieron como controles. Al mismo tiempo, se recolectaron datos sobre otros biomarcadores estándar de laboratorio y de parámetros clínicos (6). Los resultados mostraron que el VMP en la admisión y al inicio de los síntomas se asoció fuertemente con mortalidad como desenlace. De 183 pacientes con sepsis, 41 (21,5\%) de los que murieron, tenían el VMP mayor al de los supervivientes (9,6 vs 9,19 fl, respectivamente). En el momento en que los hemocultivos fueron positivos, esos valores habían aumentado (11,2 vs 9,7fl). Parámetros cómo la temperatura, el contaje de leucocitos, el lactato, la procalcitonina, y la proteína C-reactiva (PCR), por otro lado, no fueron del todo predictivos del resultado. Luego, los investigadores determinaron que el mejor predictor de muerte o de supervivencia fue cuando se utiliza un valor discriminatorio de VMP 8,7 fl.

En Ecuador Vélez et al., determinaron que el volumen medio plaquetario igual o mayor a 10 (7) y luego en un estudio con mayor número de pacientes, un VMP > 8,7 (AUC: 0,87) (8), se asoció fuertemente con incremento en la mortalidad; los estudios fueron realizados en pacientes críticos con sepsis de cualquier etiología. Fue llamativo, además, la asociación positiva entre el contaje leucocitario y volumen medio plaquetario reportado en los dos estudios.

Sánchez-Calzada et al, en un estudio prospectivo con 202 pacientes, determinaron que el VMP inferior a 7,7 fl es una herramienta útil para descartar etiología infecciosa del síndrome de respuesta inflamatoria sistémica (9).

En 2015, Ates et al, realizaron un estudio de casos y controles intentando determinar si el VMP y la razón $\mathrm{VMP} /$ contaje plaquetario podían determinar si el síndrome de respuesta inflamatoria sistémica se debía a procesos sépticos, sin éxito ya que los valores de sensibilidad y especificidad tanto del VMP y la razón $\mathrm{VMP} /$ contaje plaquetario fueron bajos y sin significancia estadística (10).

En 2016, Oh GH et al, realizaron un estudio retrospectivo en 120 pacientes adultos con diagnóstico de sepsis severa y choque séptico reanimados por una terapia guiada por objetivos y demostraron que el VMP y el contaje plaquetario por si solos, no eran buenos estimadores de mortalidad; sin embargo, la razón $\mathrm{VMP} /$ contaje plaquetario a la admisión y a las 24 horas fueron buenos predictores de mortalidad a los 28 días (>3,71 al ingreso HR: 4,274; IC del 95\%: $1,228$ a 14,$874 ; \mathrm{p}=0,023)$ y $(>6,49$ a las 24 horas HR: 2,719; IC del 95\%: 1,048-7,051; $p=0,04)(11)$.

Tajarernmuang et al, realizaron un meta-análisis de 11 estudios observacionales con 3274 pacientes, que no demostró que la del VMP inicial era un buen predictor de mortalidad; sin embargo, había mejor rendimiento de la prueba a partir del tercer día, la heterogeneidad de los estudios analizados es alta; por lo que las conclusiones son inciertas (2).

Wang et al, realizaron un estudio retrospectivo en pacientes con pancreatitis aguda, evaluaron el ancho de distribución plaquetario como factor predictor de disfunción orgánica, encontraron una sensibilidad de 0,867 , especificidad de 0,771 y un área bajo la curva (AUC) de 0,87 (12).

En 2018, Yalavarthi et al, realizaron un estudio sobre la utilidad de los índices plaquetarios (volumen medio plaquetario, contaje plaquetario, ancho de distribución) en tres tipos de pacientes, el grupo 1 incluyó la trombocitopenia inmune, el grupo 2 con trombocitopenia de causas no inmunológicas (sepsis entre otras) y el grupo 3, misceláneas; en todos los grupos de trombocitopenia debido a destrucción acelerada de plaquetas, el MPV y P-LCR fueron significativamente más altos (13).

En la tabla 1, se resume los estudios publicados sobre el volumen medio plaquetario.

\section{CONCLUSIONES}

- La plaqueta ha dejado de ser un simple fragmento celular que interviene en la coagulación.

- Su implicación en estados inflamatorios 
trombóticos ha sido ampliamente validada.

- En la sepsis, pese a ser un fenómeno inflamatorio secundario a una etiología infecciosa, su rol está en investigación. El volumen medio plaquetario y la razón entre volumen medio plaquetario/ contaje plaquetario se han convertido en importantes biomarcadores, la literatura revisada orienta a su uso, pero la validación estadística no es contundente (estudios observacionales y metaanálisis con alta heterogeneidad). Se deberá seguir estudiando la utilidad del incremento del VMP al ingreso, 48 y 72 horas y de la razón, al ingreso y a las 24 horas como predictores de mal pronóstico (mortalidad) en pacientes con severos estados sépticos.

\section{Aspectos a Futuro}

Es una línea de investigación interesante que implica un biomarcador anatómico de bajo costo y asequible, se requieren estudios con mayor número de pacientes que definan su potencial utilidad.

\section{Correspondencia:}

Jorge Luis Vélez.

Universidad Central del Ecuador-Hospital Pablo

Arturo Suárez.

Correo electrónico: jorge.velez.p@upch.pe /

jorgeluisvelez13@hotmail.com

Teléfono: 0998203672

\section{REFERENCIAS BIBLIOGRÁFICAS}

1. Kucukardali Y, Yalcin O, Hakan T. Mean Platelet Volume (MPV) in Intensive Care Unit (ICU) Patients: Is it a useful parameter in assessing prediction for mortality? Journal of Medicine and Medical Sciences. 2010; 1(3):61-64.

2. Tajarernmuang P, Phrommintikul A, Chittawatanarat $\mathrm{K}$. The role of mean platelet volume as a predictor of mortality in critically ill patients: A Systematic Review and Meta-Analysis. Critical Care Research and Practice. 2016; 2016: 0-0. doi: https://doi. org/10.1155/2016/4370834
3. Gutierrez-Romero A, Gutierrez-Grobe Y, CariiloEsper R. Volumen Plaquetario Medio: el tamaño si importa. Med Int Mex. 2013; 29:307-310.

4. Kitazawa T, Yoshino Y, Tatsuno K, Ota Y, Yotsuyanagi $\mathrm{H}$. Changes in the Mean Platelets Volume Levels after bloedstream infections have Pronostic Value. Intern Med. 2013; 52:1487-1493.

5. Pabón P, Ballesteros F, Moríniigo JL. Influencia del volumen plaquetario medio sobre el pronóstico a corto plazo del infarto agudo de miocardio. Rev Esp Cardiol. 1998; 51(10):816-22.

6. Keller D. Platelet Size Predicts Mortality in Patients with Sepsis. Medscape. 2012; https://www.medscape. com/viewarticle/767116? src $=$ trendmd_pilot

7. Vélez JL, Bucheli R, Ramírez V. Volumen medio plaquetario: predictor de mortalidad en sepsis de pacientes críticos. Revista Metrociencia. 2015;23:4043.

8. Vélez JL, Jara A, Vélez P. ¿Es el volumen medio plaquetario un predictor de mortalidad en sepsis? de pacientes críticos. Revista de la Facultad de Ciencias Médicas. 2017; 42(1);84-90.

9. Sànchez-Calzada A, Navarro JL, Delgado L. Mean platelet volume a marker of sepsis in patients admitted to intensive therapy. Intensive Care Medicine Experimental. 2015; 3(suppl 1):A871.

10. Ates S, Okzus H, Dogu B. Can mean platelet volume and mean platelet volume/platelet count ratio be used as a diagnostic marker for sepsis and systematic inflammatory response syndrome? Saudi Med J.2015;36(10):1186-90. doi: 10.15537/ smj.2015.10.10718

11. Oh GH, Chung SP, Park YS et al. Mean platelet volume to platelet count ratio as a promising predictor of early mortality in severe sepsis. Shock. 2017; 47(3):323-330. doi: 10.1097/ SHK.0000000000000718.

12. Yalavarthi S, Halder NR, Pagidikalava A. Utility of platelet indices in diagnosing the underlying cause thrombocytopenia due to accelerated platelet destruction. Annals of Pathology and Laboratory Medicine. 2018; 5(4): 0-0. doi: 10.21276/ APALM. 1717

13. Hampton T. Platelets 'role in adaptative immunity main contribute to sepsis and shock. JAMA. 2018; 319(13):1311. 